\title{
Catalytic Enantioselective O-H Insertion Reactions
}

\author{
Thomas C. Maier and Gregory C. Fu* \\ Department of Chemistry, Massachusetts Institute of Technology, Cambridge, Massachusetts \\ 02139
}

\section{Supporting Information}

\section{General}

All reactions were carried out in oven-dried glassware under an atmosphere of nitrogen or argon. 1,2-Dichloroethane was purchased from Fluka (anhydrous); other solvents were purified and dried according to generally accepted procedures. 2Trimethylsilylethanol, 2,2,2-trifluoroethanol, benzyl alcohol, $p$-methoxybenzyl alcohol, and phenol were purchased, degassed, and stored under nitrogen; all other alcohols were distilled from magnesium under an argon atmosphere. Copper(II) triflate, along with all other chemicals (including the various copper salts) were purchased, stored under inert gas, and used without further purification, unless noted otherwise. Ligand 1 was prepared according to a literature procedure. ${ }^{1}$

Mass spectrometric measurements were performed on an Agilent Technologies LC/MSD SL system (1100 Series). HPLC analyses were carried out on an Agilent 1100 Series system with Daicel Chiralpak ${ }^{\circledR}$ columns in hexanes/iso-propanol mixtures. GC analysis (chiral) was performed on a Hewlett-Packard HP 5890 Series II Plus apparatus. 


\section{Preparation of the Diazo Compounds}

General Procedure. All of the $\alpha$-diazo acetates were prepared via direct diazo transfer: Under an inert atmosphere, the $\alpha$-aryl acetate was dissolved in a small amount of $\mathrm{MeCN}$ at room temperature. DBU (1.2-5.0 equiv) was added, and then tosyl azide (1.2-4.5 equiv) was added dropwise over 30 minutes with stirring. After $12 \mathrm{~h}$, the solvent was evaporated, and the residue was dissolved in $\mathrm{Et}_{2} \mathrm{O}$ and a $5 \%$ aqueous $\mathrm{KOH}$ solution. The layers were separated, and the aqueous layer was extracted twice with $\mathrm{Et}_{2} \mathrm{O}$. The combined organic layers were dried $\left(\mathrm{MgSO}_{4}\right)$, filtered, and concentrated in vacuo. If a large excess of tosyl azide was employed in order to achieve an acceptable conversion (or if tosyl azide and the desired $\alpha$-diazo acetate have the same $R_{f}$ value in other solvent systems), the tosyl azide was removed from the crude $\alpha$-diazo acetate by chromatography on deactivated silica gel (toluene $/ \mathrm{Et}_{3} \mathrm{~N}$ as the eluent). Further purification was achieved by flash chromatography on deactivated silica gel (hexanes/EtOAc/ $\mathrm{Et}_{3} \mathrm{~N}$ as the eluent).

All of the diazo compounds, except methyl $\alpha$-diazo- $\alpha$-2-fluorophenyl-acetate and ethyl $\alpha$-diazo- $\alpha-2$-methylphenyl-acetate have previously been reported.

Ethyl $\alpha$-diazo- $\alpha$-(2-methylphenyl)acetate. The general procedure was followed, using ethyl 2-(methylphenyl)acetate (1.07 g, $6.00 \mathrm{mmol}), \mathrm{MeCN}(10 \mathrm{~mL}), \mathrm{DBU}(4.02 \mathrm{~g}$, $3.95 \mathrm{~mL}, 26.4 \mathrm{mmol})$, and tosyl azide $(4.73 \mathrm{~g}, 24.0 \mathrm{mmol})$. The excess tosyl azide was removed by flash chromatography (toluene $/ \mathrm{Et}_{3} \mathrm{~N}$ 20:0.1), and the resulting residue was purified by flash chromatography (hexanes/EtOAc/ $\mathrm{Et}_{3} \mathrm{~N}$ 20:1:0.1) to yield the title compound as a yellow oil: $0.87 \mathrm{~g}(4.26 \mathrm{mmol}, 71 \%)$.

${ }^{1} \mathrm{H} \mathrm{NMR}\left(\mathrm{CDCl}_{3}, 400 \mathrm{MHz}\right) \delta$ 7.43-7.39 (m, 1H), 7.31-7.24 (m, 3H), $4.32(\mathrm{q}, J=7.1 \mathrm{~Hz}$, $2 \mathrm{H}), 2.33(\mathrm{~s}, 3 \mathrm{H}), 1.33(\mathrm{t}, J=7.1 \mathrm{~Hz}, 3 \mathrm{H})$.

${ }^{13} \mathrm{C} \mathrm{NMR}\left(\mathrm{CDCl}_{3}, 100 \mathrm{MHz}\right) \delta 166.3,137.8,131.01,130.97,129.0,126.6,124.4,61.3,20.2$, 14.7. The resonance of the carbon that bears the diazo group was not detected.

IR (film) 3065, 2982, 2088, 1704, 1491, 1462, 1369, 1337, 1292, 1257, 1173, 1156, 1037, $756,661$.

Methyl $\alpha$-diazo- $\alpha$-(2-fluorophenyl)acetate. Under inert atmosphere methyl 2fluorophenyl-acetate $(500 \mathrm{mg}, 2.97 \mathrm{mmol})$ is dissolved in MeCN (18 ml) and 1,8diazabicyclo[5.4.0]undec-7-ene (DBU, $556 \mathrm{mg}, 546 \mu \mathrm{l}, 3.65 \mathrm{mmol}$ ) is added at room temperature. Then tosyl azide $(710 \mathrm{mg}, 3.60 \mathrm{mmol})$ is added dropwise with stirring at rt. After $20 \mathrm{~h}$ the solvent is evaporated, the residue dissolved in $\mathrm{Et}_{2} \mathrm{O}$ and $5 \%$ aqueous $\mathrm{KOH}$ solution. The layers are separated and the aqueous layer is extracted twice with $\mathrm{Et}_{2} \mathrm{O}$. The combined org. layers are dried $\left(\mathrm{MgSO}_{4}\right)$, filtered, and concentrated in vacuo. The resulting residue is purified by flash chromatography (hexanes/EtOAc $/ \mathrm{Et}_{3} \mathrm{~N}$ 19:1:0.1) to yield the title compound as a yellow oil: $524 \mathrm{mg}(2.70 \mathrm{mmol}, 91 \%)$.

${ }^{1} \mathrm{H} \mathrm{NMR}\left(\mathrm{CDCl}_{3}, 400 \mathrm{MHz}\right) \delta 7.70(\mathrm{td}, J=7.8 \mathrm{~Hz}, 1.9 \mathrm{~Hz}, 1 \mathrm{H}), 7.28-7.17(\mathrm{~m}, 2 \mathrm{H}), 7.12-$ $7.05(\mathrm{~m}, 1 \mathrm{H}), 3.84(\mathrm{~s}, 3 \mathrm{H})$. 
${ }^{13} \mathrm{C} \mathrm{NMR}\left(\mathrm{CDCl}_{3}, 100 \mathrm{MHz}\right) \delta 165.8,158.5(\mathrm{~d}, J=248 \mathrm{~Hz}), 129.6,128.8(\mathrm{~d}, J=8.3 \mathrm{~Hz})$, $124.8(\mathrm{~d}, J=3.5 \mathrm{~Hz}), 115.9(\mathrm{~d}, J=21.3 \mathrm{~Hz}), 113.9(\mathrm{~d}, J=11.9 \mathrm{~Hz}), 52.4$. The resonance of the carbon that bears the diazo group was not detected.

IR (film) 3068, 3002, 2955, 2098, 1708, 1497, 1436, 1350, 1288, 1247, 1194, 1151, 1047, $1029,756,657$. 


\section{Catalytic Enantioselective O-H Insertion Reactions}

General Procedure for Table 2. In a glove box, $\mathrm{Cu}(\mathrm{OTf})_{2}(2.9 \mathrm{mg}, 0.0080 \mathrm{mmol})$ and ligand (+)-1 (7.9 mg, $0.015 \mathrm{mmol})$ were dissolved in 1,2-dichloroethane $(1.0 \mathrm{~mL})$ that contained water (distilled and degassed; $0.016 \mathrm{mmol}$ ). The resulting mixture was stirred for $20 \mathrm{~min}$ at rt, and then it was passed through an acrodisc filter and added in one portion to a solution of the diazo compound $(0.40 \mathrm{mmol})$ and the alcohol $(0.42 \mathrm{mmol})$ in 1,2-dichloroethane $(40 \mathrm{~mL})$. The reaction mixture was stirred for $1.0 \mathrm{~h}$ at $\mathrm{rt}$. Then, the solvent was evaporated, and the residue was purified by flash chromatography (hexane/EtOAc or pentane $/ \mathrm{Et}_{2} \mathrm{O}$ as the eluent).

The ee values were determined via HPLC on Daicel Chiralpak columns or via GC.

If removal of the trimethylsilylethyl group was necessary for ee analysis, an aliquot of the product was dissolved in $\mathrm{CH}_{2} \mathrm{Cl}_{2}$ and treated with an excess of $\mathrm{BF}_{3} \cdot \mathrm{OEt}_{2}$ for $15 \mathrm{~min}$ at rt. The reaction was quenched with a solution of sat. $\mathrm{NaHCO}_{3}$, and the organic layer was dried $\left(\mathrm{MgSO}_{4}\right)$, filtered, concentrated, and subjected to HPLC analysis.

If reduction of the ester was necessary for ee analysis, an aliquot of the product was dissolved in $\mathrm{Et}_{2} \mathrm{O}$ and treated with an excess of $\mathrm{LiAlH}_{4}$ at $\mathrm{rt}$ for $45 \mathrm{~min}$. The reaction was quenched with a solution of $5 \% \mathrm{H}_{2} \mathrm{SO}_{4}$, and the organic layer was dried $\left(\mathrm{MgSO}_{4}\right)$, filtered, concentrated, and subjected to HPLC analysis.

The second runs were performed with (-)-1.

Methyl $\alpha$-phenyl- $\alpha$-methoxy-acetate (Table 2, entry 1) [26164-27-2 (S)]. The compound was prepared according to the General Procedure using methanol (13.5 mg, $17.1 \mu \mathrm{L}$ ). After chromatography on silica gel (pentane $/ \mathrm{Et}_{2} \mathrm{O} 15: 1$ ), the title compound was isolated as a colorless oil: run 1: $61 \mathrm{mg}(85 \%$; $72 \%$ ee); run 2: $62 \mathrm{mg}(86 \%$; 66\% ee). The ee was determined via HPLC on an OD-H column (hexanes / iso-propanol 99:1, flow $1.0 \mathrm{~mL} / \mathrm{min}$ ) with $\mathrm{t}_{\mathrm{r}}$ (major) $8.4 \mathrm{~min}, \mathrm{t}_{\mathrm{r}}$ (minor) $17.7 \mathrm{~min}$.

$[\alpha]^{23}=94.3\left(\mathrm{c}=1.01, \mathrm{CHCl}_{3}\right)$.

(S)-(+)-Methyl $\alpha$-phenyl- $\alpha$-ethoxy-acetate (Table 2, entry 2) [65792-30-5]. The compound was prepared according to the General Procedure using ethanol (19.4 mg, $24.5 \mu \mathrm{L}$ ). After chromatography on silica gel (hexanes $/ \mathrm{Et}_{2} \mathrm{O} 18: 1$ ), the title compound was isolated as a colorless oil: run 1: $65 \mathrm{mg}(84 \% ; 86 \%$ ee); run 2: $66 \mathrm{mg}(85 \% ; 87 \%$ ee). The ee was determined via GC on a G-TA column with $\mathrm{t}_{\mathrm{r}}$ (minor) $14.89 \mathrm{~min}, \mathrm{t}_{\mathrm{r}}$ (major) $16.02 \mathrm{~min}$.

$[\alpha]^{23}=87.2\left(\mathrm{c}=1.00, \mathrm{CHCl}_{3}\right)$. The absolute configuration was determined by comparison with authentic material prepared by ethylation of commercially available mandelic mandelate.

Methyl $\alpha$-phenyl- $\alpha-1-$ methylethoxy-acetate (Table 2, entry 3) [130115-06-9 (R)]. The compound was prepared according to the General Procedure using isopropanol $(25.3 \mathrm{mg}, 32.2 \mu \mathrm{L})$. After chromatography on silica gel (pentane $/ \mathrm{Et}_{2} \mathrm{O} 20: 1$ ), the title compound was isolated as a colorless oil: run 1: $61 \mathrm{mg}$ (73\%; 69\% ee); run 2: $65 \mathrm{mg}$ (78\%; 
$67 \%$ ee). The ee was determined via GC on a G-TA column with $\mathrm{t}_{\mathrm{r}}$ (minor) $30.52 \mathrm{~min}$, $\mathrm{t}_{\mathrm{r}}$ (major) $33.20 \mathrm{~min}$.

$$
[\alpha]^{23}=60.4\left(\mathrm{c}=1.00, \mathrm{CHCl}_{3}\right) \text {. }
$$

Methyl $\alpha$-phenyl- $\alpha-1,1$-dimethylethoxy-acetate (Table 2, entry 4). The General Procedure was followed, using tert-butanol ( $31.1 \mathrm{mg}, 40.2 \mu \mathrm{L})$. None of the title compound could be isolated (a trace was formed, according to ${ }^{1} \mathrm{H}$ NMR spectroscopy).

(S)-(+)-Methyl $\alpha$-phenyl- $\alpha$-2-trimethylsilylethoxy-acetate (Table 2, entry 5; Table 3, entry 1). The compound was prepared according to the General Procedure using 2trimethylsilylethanol $(49.7 \mathrm{mg}, 60.2 \mu \mathrm{L})$. After chromatography on silica gel (hexanes/EtOAc 20:1), the title compound was isolated as a colorless oil: run 1: $100 \mathrm{mg}$ (94\%; 90\% ee); run 2: $99 \mathrm{mg}$ (93\%; 89\% ee). The ee was determined after cleavage of the trimethylsilylethyl group via HPLC on an OD-H column (hexanes/iso-propanol 90:10, flow $1.0 \mathrm{~mL} / \mathrm{min}$ ) with $\mathrm{t}_{\mathrm{r}}$ (major) $8.3 \mathrm{~min}, \mathrm{t}_{\mathrm{r}}$ (minor) $13.9 \mathrm{~min}$.

$[\alpha]_{D}^{23}=68.3\left(\mathrm{c}=1.01, \mathrm{CHCl}_{3}\right)$. The absolute configuration was determined by deprotection and comparison with commercially available methyl mandelate.

${ }^{1} \mathrm{H}$ NMR $\left(\mathrm{CDCl}_{3}, 400 \mathrm{MHz}\right)$ 8 7.48-7.44 (m, 2H), 7.39-7.21 (m, 3H), $4.89(\mathrm{~s}, 1 \mathrm{H}), 3.72(\mathrm{~s}$, $3 \mathrm{H}), 3.65(\mathrm{ddd}, J=10.2 \mathrm{~Hz}, 9.2 \mathrm{~Hz}, 6.4 \mathrm{~Hz}, 1 \mathrm{H}), 3.55(\mathrm{ddd}, J=10.2 \mathrm{~Hz}, 9.2 \mathrm{~Hz}, 6.2 \mathrm{~Hz}$, 1H), 1.07 (ddd, $J=13.9 \mathrm{~Hz}, 10.2 \mathrm{~Hz}, 6.4 \mathrm{~Hz}, 1 \mathrm{H}), 1.02(\mathrm{ddd}, J=13.9 \mathrm{~Hz}, 10.2 \mathrm{~Hz}, 6.2 \mathrm{~Hz}$, $1 \mathrm{H}), 0.01(\mathrm{~s}, 9 \mathrm{H})$.

${ }^{13} \mathrm{C} \mathrm{NMR}\left(\mathrm{CDCl}_{3}, 100 \mathrm{MHz}\right) \delta 171.8,136.9,128.8,128.7,127.3,80.8,67.5,52.4,18.4,-$ 1.2.

IR (film) 3066, 3033, 2953, 2894, 1755, 1455, 1435, 1249, 1208, 1171, 1117, 860, 839, 729, $697 \mathrm{~cm}^{-1}$.

MS (ESI) calcd for $\mathrm{C}_{14} \mathrm{H}_{22} \mathrm{NaO}_{3} \mathrm{Si}\left(\mathrm{M}^{+}+\mathrm{Na}\right)$ 289.1, found 289.0.

Methyl $\alpha$-phenyl- $\alpha-2,2,2$-trifluoroethoxy-acetate (Table 2, entry 6). The General Procedure was followed, using 2,2,2-trifluoroethanol $(42.0 \mathrm{mg}, 30.6 \mu \mathrm{L})$. None of the title compound could be isolated (a trace was formed, according to ${ }^{1} \mathrm{H}$ NMR spectroscopy).

Methyl $\alpha$-phenyl- $\alpha$-benzyloxy-acetate (Table 2, entry 7) [60300-84-7]. The compound was prepared according to the General Procedure using benzyl alcohol (45.4 $\mathrm{mg}$, $43.5 \mu \mathrm{L}$ ). After chromatography on silica gel (hexanes $/ \mathrm{Et}_{2} \mathrm{O} 12: 1$ ), the title compound was isolated as a colorless oil: run 1: $87 \mathrm{mg}$ (85\%; 76\% ee); run 2: $89 \mathrm{mg}$ (87\%; $77 \%$ ee). The ee was determined after reduction of the ester via HPLC on an AS-H column (hexanes / iso-propanol 95:5, flow $1.0 \mathrm{~mL} / \mathrm{min}$ ) with $\mathrm{t}_{\mathrm{r}}$ (major) $9.3 \mathrm{~min}, \mathrm{t}_{\mathrm{r}}($ minor) $10.4 \mathrm{~min}$.

$[\alpha]_{D}^{23}=77.7\left(\mathrm{c}=1.00, \mathrm{CHCl}_{3}\right)$. The absolute configuration was assigned by comparison with literature data. ${ }^{2}$

Methyl $\alpha$-phenyl- $\alpha$-(4-methoxybenzyloxy)acetate (Table 2, entry 8) [641609-73-6 (S)]. The compound was prepared according to the General Procedure using $p$ - 
methoxybenzyl alcohol (58.0 mg, $52.4 \mu \mathrm{L}$ ). After chromatography on silica gel (hexanes/EtOAc 18:1), the title compound was isolated as a colorless oil: run 1: $100 \mathrm{mg}$ (87\%; 81\% ee); run 2: $100 \mathrm{mg}(87 \% ; 82 \%$ ee). The ee was determined via HPLC on an OD-H column (hexanes/iso-propanol 99:1, flow $1.0 \mathrm{~mL} / \mathrm{min}$ ) with $\mathrm{t}_{\mathrm{r}}$ (major) $12.9 \mathrm{~min}$, $\mathrm{t}_{\mathrm{r}}$ (minor) $16.3 \mathrm{~min}$.

$[\alpha]_{D}^{23}=79.4\left(\mathrm{c}=1.00, \mathrm{CHCl}_{3}\right)$. The absolute configuration was assigned by comparison with literature data. ${ }^{3}$

Methyl $\alpha$-phenyl- $\alpha$-allyloxy-acetate (Table 2, entry 9) [251934-74-4 (S)]. The compound was prepared according to the General Procedure using allyl alcohol (24.4 $\mathrm{mg}$, $28.6 \mu \mathrm{L}$ ). After chromatography on silica gel (hexanes $/ \mathrm{Et}_{2} \mathrm{O} 20: 1$ ), the title compound was isolated as a colorless oil: run 1: $64 \mathrm{mg}$ (78\%; 25\% ee); run 2: $62 \mathrm{mg}$ (75\%; $29 \%$ ee). The ee was determined via GC on a G-TA column with $t_{r}$ (minor) 20.09 min, $t_{r}$ (major) $21.52 \mathrm{~min}$.

$$
[\alpha]^{23}=25.2\left(\mathrm{c}=1.00, \mathrm{CHCl}_{3}\right) \text {. }
$$

Methyl $\alpha$-phenyl- $\alpha$-phenyloxy-acetate (Table 2, entry 10) [729589-62-2 (S)]. The compound was prepared according to the General Procedure using phenol (39.5 mg). After chromatography on silica gel (hexanes/EtOAc 18:1), the title compound was isolated as a colorless oil: run 1: $49 \mathrm{mg}(51 \%$; $11 \%$ ee); run 2: $58 \mathrm{mg}(60 \% ; 11 \%$ ee). The ee was determined via HPLC on an OD-H column (hexanes / iso-propanol 97:3, flow 1.2 $\mathrm{mL} / \mathrm{min}$ ) with $\mathrm{t}_{\mathrm{r}}$ (minor) $6.7 \mathrm{~min}, \mathrm{t}_{\mathrm{r}}$ (major) $16.2 \mathrm{~min}$.

$[\alpha]_{D}^{23}=-7.7\left(\mathrm{c}=0.98, \mathrm{CHCl}_{3}\right)$. The absolute configuration was assigned by comparison with literature data. ${ }^{4}$

Eq 2. $\mathrm{BF}_{3} \cdot \mathrm{OEt}_{2}(200 \mu \mathrm{L}, 227 \mathrm{mg}, 1.60 \mathrm{mmol})$ was added to a solution of methyl $\alpha-$ phenyl- $\alpha$-(2-trimethylsilylethoxy)acetate $(106 \mathrm{mg}, 0.40 \mathrm{mmol})$ in $\mathrm{CH}_{2} \mathrm{Cl}_{2}(8 \mathrm{~mL})$. The mixture was stirred at room temperature for $40 \mathrm{~min}$, and then the reaction was quenched with a solution of sat. $\mathrm{NaHCO}_{3}$. The organic layer was separated, and the aqueous layer was extracted with $\mathrm{CH}_{2} \mathrm{Cl}_{2}$. The combined organic layers were dried $\left(\mathrm{MgSO}_{4}\right)$, filtered, and concentrated. The residue was purified by flash chromatography on silica gel (hexanes/EtOAc 3:1), which furnished methyl mandelate as a colorless oil: $65 \mathrm{mg}(98 \%)$.

General Procedure for Table 3. In a glove box, $\mathrm{Cu}(\mathrm{OTf})_{2}(2.9 \mathrm{mg}, 0.0080 \mathrm{mmol})$ and ligand (+)-1 (7.9 mg, $0.015 \mathrm{mmol})$ were dissolved in 1,2-dichloroethane $(1.0 \mathrm{~mL})$ that contained water (distilled and degassed; $0.016 \mathrm{mmol}$ ). The resulting mixture was stirred for $20 \mathrm{~min}$ at $\mathrm{rt}$, and then it was passed through an acrodisc filter and added in one portion to a solution of the diazo compound $(0.40 \mathrm{mmol})$ and 2-trimethylsilylethanol $(49.7 \mathrm{mg}, 60.2 \mu \mathrm{L}, 0.42 \mathrm{mmol})$ in 1,2-dichloroethane $(40 \mathrm{~mL})$. The reaction mixture was stirred for $1.0 \mathrm{~h}$ at rt. Then, the solvent was evaporated, and the residue was purified by flash chromatography (hexane/EtOAc as the eluent).

The ee values were determined via HPLC on Daicel Chiralpak columns. 
If removal of the trimethylsilylethyl group was necessary for ee analysis, an aliquot of the product was dissolved in $\mathrm{CH}_{2} \mathrm{Cl}_{2}$ and treated with an excess of $\mathrm{BF}_{3} \cdot \mathrm{OEt}_{2}$ for $15 \mathrm{~min}$ at rt. The reaction was quenched with a solution of sat. $\mathrm{NaHCO}_{3}$, and the organic layer was dried $\left(\mathrm{MgSO}_{4}\right)$, filtered, concentrated, and subjected to HPLC analysis.

If reduction of the ester was necessary for ee analysis, an aliquot of the product was dissolved in $\mathrm{Et}_{2} \mathrm{O}$ and treated with an excess of $\mathrm{LiAlH}_{4}$ at $\mathrm{rt}$ for $45 \mathrm{~min}$. The reaction was quenched with a solution of $5 \% \mathrm{H}_{2} \mathrm{SO}_{4}$, and the organic layer was dried $\left(\mathrm{MgSO}_{4}\right)$,

filtered, concentrated, and subjected to HPLC analysis.

The second runs were performed with (-)-1.

(S)-(+)-Methyl $\alpha$-phenyl- $\alpha$-(2-trimethylsilylethoxy)acetate (Table 3, entry 1); see above (Table 2, entry 5 ).

Methyl $\alpha$-2-methoxyphenyl- $\alpha$-(2-trimethylsilylethoxy)acetate (Table 3, entry 2). The compound was prepared according to the General Procedure using methyl $\alpha$-diazo$\alpha$-2-(methoxyphenyl)acetate $(82.5 \mathrm{mg})$. After chromatography on silica gel (hexanes/EtOAc 15:1), the title compound was isolated as a colorless oil: run 1: $108 \mathrm{mg}$ (91\%; 97\% ee); run 2: $106 \mathrm{mg}$ (89\%; 94\% ee).

The ee was determined after reduction of the ester via HPLC on an OD-H column (hexanes / iso-propanol 95:5, flow $1.0 \mathrm{~mL} / \mathrm{min}$ ) with $\mathrm{t}_{\mathrm{r}}$ (major) $5.7 \mathrm{~min}, \mathrm{t}_{\mathrm{r}}($ minor) $7.4 \mathrm{~min}$.

$[\alpha]^{23}=80.1\left(\mathrm{c}=1.00, \mathrm{CHCl}_{3}\right)$.

${ }^{1} \mathrm{H}$ NMR $\left(\mathrm{CDCl}_{3}, 400 \mathrm{MHz}\right) \delta 7.43(\mathrm{dd}, J=7.5 \mathrm{~Hz}, 1.8 \mathrm{~Hz}, 1 \mathrm{H}), 7.31(\mathrm{ddd}, J=8.3 \mathrm{~Hz}$, $7.5 \mathrm{~Hz}, 1.8 \mathrm{~Hz}, 1 \mathrm{H}), 6.98(\mathrm{ddd}, J=7.5 \mathrm{~Hz}, 7.5 \mathrm{~Hz}, 1.1 \mathrm{~Hz}, 1 \mathrm{H}), 6.90(\mathrm{dd}, J=8.3 \mathrm{~Hz}, 1.1 \mathrm{~Hz}$, $1 \mathrm{H}), 5.32(\mathrm{~s}, 1 \mathrm{H}), 3.86(\mathrm{~s}, 3 \mathrm{H}), 3.71(\mathrm{~s}, 3 \mathrm{H}), 3.66(\mathrm{ddd}, J=10.4 \mathrm{~Hz}, 9.3 \mathrm{~Hz}, 6.2 \mathrm{~Hz}, 1 \mathrm{H}), 3.56$ $(\mathrm{ddd}, J=10.4 \mathrm{~Hz}, 9.3 \mathrm{~Hz}, 6.2 \mathrm{~Hz}, 1 \mathrm{H}), 1.05(\mathrm{ddd}, J=13.8 \mathrm{~Hz}, 10.4 \mathrm{~Hz}, 6.2 \mathrm{~Hz}, 1 \mathrm{H}), 1.01$ (ddd, $J=13.8 \mathrm{~Hz}, 10.4 \mathrm{~Hz}, 6.2 \mathrm{~Hz}, 1 \mathrm{H}), 0.00$ (s, 9H).

${ }^{13} \mathrm{C} \mathrm{NMR}\left(\mathrm{CDCl}_{3}, 100 \mathrm{MHz}\right) \delta$ 172.1, 157.1, 129.9, 128.6, 125.7, 121.0, 111.0, 74.4, 67.5, $55.9,52.3,18.3,-1.2$.

IR (film) 3002, 2952, 2896, 1751, 1602, 1494, 1465, 1249, 1204, 1094, 1029, 860, 839, 755 $\mathrm{cm}^{-1}$.

MS (ESI) calcd for $\mathrm{C}_{15} \mathrm{H}_{24} \mathrm{NaO}_{4} \mathrm{Si}\left(\mathrm{M}^{+}+\mathrm{Na}\right)$ 319.1, found 319.0.

Ethyl $\alpha$-2-methylphenyl- $\alpha$-(2-trimethylsilylethoxy)acetate (Table 3, entry 3). The compound was prepared according to the General Procedure using methyl $\alpha$-diazo- $\alpha-2-$ (methylphenyl)acetate $(81.7 \mathrm{mg})$. After chromatography on silica gel (hexanes / EtOAc $20: 1)$, the title compound was isolated as a colorless oil: run 1: $111 \mathrm{mg}(94 \% ; 80 \%$ ee); run 2: $111 \mathrm{mg}$ (94\%; 78\% ee).

The ee was determined via HPLC on an AD-H column (hexanes/iso-propanol 99:1, flow $1.0 \mathrm{~mL} / \mathrm{min}$ ) with $\mathrm{t}_{\mathrm{r}}$ (major) $4.1 \mathrm{~min}, \mathrm{t}_{\mathrm{r}}($ minor $) 5.0 \mathrm{~min}$.

$[\alpha]_{\mathrm{D}}^{23}=72.6\left(\mathrm{c}=1.00, \mathrm{CHCl}_{3}\right)$.

${ }^{1} \mathrm{H}$ NMR $\left(\mathrm{CDCl}_{3}, 400 \mathrm{MHz}\right) \delta$ 7.48-7.43 (m, 1H), 7.24-7.15 (m, 3H), $5.08(\mathrm{~s}, 1 \mathrm{H}), 4.21$ $(\mathrm{dq}, J=10.8 \mathrm{~Hz}, 7.1 \mathrm{~Hz}, 1 \mathrm{H}), 4.16(\mathrm{dq}, J=10.8 \mathrm{~Hz}, 7.1 \mathrm{~Hz}, 1 \mathrm{H}), 3.65(\mathrm{ddd}, J=10.3 \mathrm{~Hz}, 9.2$ $\mathrm{Hz}, 6.3 \mathrm{~Hz}, 1 \mathrm{H}), 3.54(\mathrm{ddd}, J=10.3 \mathrm{~Hz}, 9.2 \mathrm{~Hz}, 6.1 \mathrm{~Hz}, 1 \mathrm{H}), 2.44(\mathrm{~s}, 3 \mathrm{H}), 1.22(\mathrm{t}, J=7.1 \mathrm{~Hz}$, 
3H), $1.06(\mathrm{ddd}, J=13.9 \mathrm{~Hz}, 10.3 \mathrm{~Hz}, 6.3 \mathrm{~Hz}, 1 \mathrm{H}), 1.02(\mathrm{ddd}, J=13.9 \mathrm{~Hz}, 10.3 \mathrm{~Hz}, 6.1 \mathrm{~Hz}$, $1 \mathrm{H}), 0.02(\mathrm{~s}, 9 \mathrm{H})$.

${ }^{13} \mathrm{C} \mathrm{NMR}\left(\mathrm{CDCl}_{3}, 100 \mathrm{MHz}\right) \delta 171.5,136.4,135.6,130.7,128.5,127.6,126.4,77.9,67.4$, $61.2,19.6,18.4,14.3,-1.2$.

IR (film) 3025, 2954, 2897, 1751, 1464, 1391, 1249, 1094, 1029, 860, 839, $742 \mathrm{~cm}^{-1}$.

MS (ESI) calcd for $\mathrm{C}_{16} \mathrm{H}_{26} \mathrm{NaO}_{3} \mathrm{Si}\left(\mathrm{M}^{+}+\mathrm{Na}\right)$ 317.2, found 317.1.

Methyl $\alpha$-2-chlorophenyl- $\alpha$-(2-trimethylsilylethoxy)acetate (Table 3, entry 4). The compound was prepared according to the General Procedure using methyl $\alpha$-diazo- $\alpha-2-$ (chlorophenyl)acetate $(84.3 \mathrm{mg})$. After chromatography on silica gel (hexanes / EtOAc 20:1), the title compound was isolated as a colorless oil: run 1: $115 \mathrm{mg}(96 \% ; 95 \%$ ee); run 2: $115 \mathrm{mg}$ (96\%; 96\% ee).

The ee was determined after cleavage of the trimethylsilylethyl group via HPLC on an OD-H column (hexanes/iso-propanol 90:10, flow $1.0 \mathrm{~mL} / \mathrm{min}$ ) with $\mathrm{t}_{\mathrm{r}}$ (major) $8.1 \mathrm{~min}$, $\mathrm{t}_{\mathrm{r}}$ (minor) $9.2 \mathrm{~min}$.

$[\alpha]^{23}=82.7\left(\mathrm{c}=1.01, \mathrm{CHCl}_{3}\right)$.

${ }^{1} \mathrm{H}$ NMR $\left(\mathrm{CDCl}_{3}, 400 \mathrm{MHz}\right)$ 8 7.56-7.53 (m, 1H), 7.40-7.37 (m, 1H), 7.32-7.25 (m, 2H), $5.38(\mathrm{~s}, 1 \mathrm{H}), 3.73(\mathrm{~s}, 3 \mathrm{H}), 3.69(\mathrm{ddd}, J=10.4 \mathrm{~Hz}, 9.3 \mathrm{~Hz}, 6.2 \mathrm{~Hz}, 1 \mathrm{H}), 3.57(\mathrm{ddd}, J=10.5$ $\mathrm{Hz}, 9.3 \mathrm{~Hz}, 6.1 \mathrm{~Hz}, 1 \mathrm{H}), 1.07$ (ddd, $J=13.8 \mathrm{~Hz}, 10.5 \mathrm{~Hz}, 6.2 \mathrm{~Hz}, 1 \mathrm{H}), 1.01$ (ddd, $J=13.8$ $\mathrm{Hz}, 10.4 \mathrm{~Hz}, 6.1 \mathrm{~Hz}, 1 \mathrm{H}), 0.01(\mathrm{~s}, 9 \mathrm{H})$.

${ }^{13} \mathrm{C} \mathrm{NMR}\left(\mathrm{CDCl}_{3}, 100 \mathrm{MHz}\right) \delta 171.1,135.1,133.8,129.9,129.7,128.9,127.4,76.9,67.9$, $52.5,18.4,-1.2$.

IR (film) 3068, 2953, 2896, 1754, 1474, 1436, 1250, 1209, 1106, 860, $839 \mathrm{~cm}^{-1}$.

MS (ESI) calcd for $\mathrm{C}_{14} \mathrm{H}_{21} \mathrm{ClNaO}_{3} \mathrm{Si}\left(\mathrm{M}^{+}+\mathrm{Na}\right)$ 323.1, found 323.0.

Methyl $\alpha$-2-fluorophenyl- $\alpha$-(2-trimethylsilylethoxy)acetate (Table 3, entry 5). The compound was prepared according to the General Procedure using methyl $\alpha$-diazo- $\alpha-2-$ (fluorophenyl)acetate $(77.7 \mathrm{mg})$. After chromatography on silica gel (hexanes / EtOAc 18:1), the title compound was isolated as a colorless oil: run 1: $111 \mathrm{mg}(98 \% ; 97 \%$ ee); run 2: $110 \mathrm{mg}$ (97\%; $98 \%$ ee).

The ee was determined after cleavage of the trimethylsilylethyl group via HPLC on an OD-H column (hexanes/iso-propanol 90:10, flow $1.0 \mathrm{~mL} / \mathrm{min}$ ) with $\mathrm{t}_{\mathrm{r}}$ (major) $7.7 \mathrm{~min}$, $\mathrm{t}_{\mathrm{r}}(\mathrm{minor}) 9.0 \mathrm{~min}$.

$[\alpha]^{23}=87.0\left(\mathrm{c}=1.02, \mathrm{CHCl}_{3}\right)$.

${ }^{1} \mathrm{H}$ NMR $\left(\mathrm{CDCl}_{3}, 400 \mathrm{MHz}\right) \delta$ 7.52-7.47 (m, 1H), 7.35-7.29 (m, 1H), 7.19-7.14 (m, 1H), 7.10-7.05 (m, 1H), $5.24(\mathrm{~s}, 1 \mathrm{H}), 3.73(\mathrm{~s}, 3 \mathrm{H}), 3.69(\mathrm{ddd}, J=10.3 \mathrm{~Hz}, 9.2 \mathrm{~Hz}, 6.2 \mathrm{~Hz}, 1 \mathrm{H})$, $3.56(\mathrm{ddd}, J=10.4 \mathrm{~Hz}, 9.2 \mathrm{~Hz}, 6.1 \mathrm{~Hz}, 1 \mathrm{H}), 1.06(\mathrm{ddd}, J=13.8 \mathrm{~Hz}, 10.3 \mathrm{~Hz}, 6.2 \mathrm{~Hz}, 1 \mathrm{H})$, $1.00(\mathrm{ddd}, J=13.8 \mathrm{~Hz}, 10.4 \mathrm{~Hz}, 6.1 \mathrm{~Hz}, 1 \mathrm{H}), 0.01(\mathrm{~s}, 9 \mathrm{H})$.

${ }^{13} \mathrm{C} \mathrm{NMR}\left(\mathrm{CDCl}_{3}, 100 \mathrm{MHz}\right) \delta 171.2,160.5(\mathrm{~d}, J=248 \mathrm{~Hz}), 130.4(\mathrm{~d}, J=8.1 \mathrm{~Hz}), 128.9$ $(\mathrm{d}, J=3.2 \mathrm{~Hz}), 124.7(\mathrm{~d}, J=3.7 \mathrm{~Hz}), 124.5(\mathrm{~d}, J=14.4 \mathrm{~Hz}), 115.7(\mathrm{~d}, J=21.8 \mathrm{~Hz}), 73.6(\mathrm{~d}, J$ $=3.0 \mathrm{~Hz}), 67.8,52.5,18.3,-1.2$.

IR (film) 3052, 2954, 2896, 1755, 1491, 1458, 1249, 1207, 1114, 860, 839, $757 \mathrm{~cm}^{-1}$.

MS (ESI) calcd for $\mathrm{C}_{14} \mathrm{H}_{21} \mathrm{FNaO}_{3} \mathrm{Si}\left(\mathrm{M}^{+}+\mathrm{Na}\right)$ 307.1, found 307.0. 
Ethyl $\alpha$-3-methoxyphenyl- $\alpha$-(2-trimethylsilylethoxy)acetate (Table 3, entry 6). The compound was prepared according to the General Procedure using ethyl $\alpha$-diazo- $\alpha-3-$ (methoxyphenyl)acetate $(88.1 \mathrm{mg}$ ). After chromatography on silica gel (hexanes/EtOAc 18:1), the title compound was isolated as a colorless oil: run 1: $119 \mathrm{mg}(97 \% ; 90 \%$ ee); run 2: $118 \mathrm{mg}$ (95\%; 88\% ee).

The ee was determined via HPLC on an AS-H column (hexanes / iso-propanol 99.8:0.2, flow $0.8 \mathrm{~mL} / \mathrm{min}$ ) with $\mathrm{t}_{\mathrm{r}}\left(\right.$ major) $6.6 \mathrm{~min}, \mathrm{t}_{\mathrm{r}}(\mathrm{minor}) 7.1 \mathrm{~min}$.

$[\alpha]^{23}=49.9\left(\mathrm{c}=0.98, \mathrm{CHCl}_{3}\right)$.

${ }^{1} \mathrm{H}$ NMR $\left(\mathrm{CDCl}_{3}, 400 \mathrm{MHz}\right)$ 8 7.29-7.24 (m, 1H), 7.05-7.02 (m, 2H), 6.88-6.85 (m, $\left.1 \mathrm{H}\right)$, $4.84(\mathrm{~s}, 1 \mathrm{H}), 4.21(\mathrm{dq}, J=10.8 \mathrm{~Hz}, 7.1 \mathrm{~Hz}, 1 \mathrm{H}), 4.16(\mathrm{dq}, J=10.8 \mathrm{~Hz}, 7.1 \mathrm{~Hz}, 1 \mathrm{H}), 3.81(\mathrm{~s}$, $3 \mathrm{H}), 3.65(\mathrm{ddd}, J=9.9 \mathrm{~Hz}, 9.2 \mathrm{~Hz}, 6.5 \mathrm{~Hz}, 1 \mathrm{H}), 3.56(\mathrm{ddd}, J=10.0 \mathrm{~Hz}, 9.2 \mathrm{~Hz}, 6.3 \mathrm{~Hz}, 1 \mathrm{H})$, $1.23(\mathrm{t}, J=7.1 \mathrm{~Hz}, 3 \mathrm{H}), 1.07(\mathrm{ddd}, J=13.9 \mathrm{~Hz}, 10.0 \mathrm{~Hz}, 6.5 \mathrm{~Hz}, 1 \mathrm{H}), 1.02(\mathrm{ddd}, J=13.9 \mathrm{~Hz}$, $9.9 \mathrm{~Hz}, 6.3 \mathrm{~Hz}, 1 \mathrm{H}), 0.02(\mathrm{~s}, 9 \mathrm{H})$.

${ }^{13} \mathrm{C} \mathrm{NMR}\left(\mathrm{CDCl}_{3}, 100 \mathrm{MHz}\right) \delta$ 171.3, 159.9, 138.6, 129.7, 119.7, 114.6, 112.3, 80.8, 67.4, $61.3,55.5,18.3,14.3,-1.2$.

IR (film) 2954, 2896, 1751, 1601, 1490, 1250, 1183, 1156, 1114, 1044, 860, $839 \mathrm{~cm}^{-1}$.

MS (ESI) calcd for $\mathrm{C}_{16} \mathrm{H}_{26} \mathrm{NaO}_{4} \mathrm{Si}\left(\mathrm{M}^{+}+\mathrm{Na}\right)$ 333.2, found 333.0.

Methyl $\alpha$-3-chlorophenyl- $\alpha$-(2-trimethylsilylethoxy)acetate (Table 3, entry 7). The compound was prepared according to the General Procedure using methyl $\alpha$-diazo- $\alpha-3-$ (chlorophenyl)acetate $(84.3 \mathrm{mg})$. After chromatography on silica gel (hexanes/EtOAc 20:1), the title compound was isolated as a colorless oil: run 1: $112 \mathrm{mg}(93 \% ; 64 \%$ ee); run 2: $109 \mathrm{mg}$ (91\%; 65\% ee).

The ee was determined after cleavage of the trimethylsilylethyl group via HPLC on an OD-H column (hexanes/iso-propanol 90:10, flow $1.0 \mathrm{~mL} / \mathrm{min}$ ) with $\mathrm{t}_{\mathrm{r}}($ major) $7.6 \mathrm{~min}$, $\mathrm{t}_{\mathrm{r}}(\mathrm{minor}) 9.1 \mathrm{~min}$.

$[\alpha]^{23}=43.1\left(\mathrm{c}=1.02, \mathrm{CHCl}_{3}\right)$.

${ }^{1} \mathrm{H}$ NMR $\left(\mathrm{CDCl}_{3}, 400 \mathrm{MHz}\right)$ 8 7.48-7.46 (m, 1H), 7.36-7.28 (m, 3H), $4.85(\mathrm{~s}, 1 \mathrm{H}), 3.73(\mathrm{~s}$, $3 \mathrm{H}), 3.65(\mathrm{ddd}, J=10.2 \mathrm{~Hz}, 9.1 \mathrm{~Hz}, 6.4 \mathrm{~Hz}, 1 \mathrm{H}), 3.54(\mathrm{ddd}, J=10.3 \mathrm{~Hz}, 9.1 \mathrm{~Hz}, 6.2 \mathrm{~Hz}$, 1H), $1.07(\mathrm{ddd}, J=13.9 \mathrm{~Hz}, 10.3 \mathrm{~Hz}, 6.4 \mathrm{~Hz}, 1 \mathrm{H}), 1.02(\mathrm{ddd}, J=13.9 \mathrm{~Hz}, 10.2 \mathrm{~Hz}, 6.2 \mathrm{~Hz}$, $1 \mathrm{H}), 0.02(\mathrm{~s}, 9 \mathrm{H})$.

${ }^{13} \mathrm{C} \mathrm{NMR}\left(\mathrm{CDCl}_{3}, 100 \mathrm{MHz}\right) \delta$ 171.3, 138.9, 134.7, 130.0, 128.9, 127.4, 125.4, 80.1, 67.8, $52.6,18.4,-1.2$.

IR (film) 3058, 2954, 2895, 1756, 1434, 1250, 1208, 1173, 1118, 860, $839 \mathrm{~cm}^{-1}$.

MS (ESI) calcd for $\mathrm{C}_{14} \mathrm{H}_{21} \mathrm{ClNaO}_{3} \mathrm{Si}\left(\mathrm{M}^{+}+\mathrm{Na}\right)$ 323.1, found 323.0.

Methyl $\alpha$-4-methoxyphenyl- $\alpha$-(2-trimethylsilylethoxy)acetate (Table 3, entry 8). The compound was prepared according to the General Procedure using methyl $\alpha$-diazo$\alpha-4$-(methoxyphenyl)acetate $(82.5 \mathrm{mg})$. After chromatography on silica gel (hexanes/EtOAc 18:1), the title compound was isolated as a colorless oil: run 1: $102 \mathrm{mg}$ (86\%; 87\% ee); run 2: $99 \mathrm{mg}(83 \%$; $84 \%$ ee).

The ee was determined after reduction of the ester via HPLC on an OD-H column (hexanes / iso-propanol 99.4:0.6, flow $1.2 \mathrm{~mL} / \mathrm{min}$ ) with $\mathrm{t}_{\mathrm{r}}$ (minor) $10.2 \mathrm{~min}$, $\mathrm{t}_{\mathrm{r}}$ (major) 11.4 $\min$. 
$[\alpha]^{23}=81.3\left(\mathrm{c}=0.99, \mathrm{CHCl}_{3}\right)$.

${ }^{1} \mathrm{H} \mathrm{NMR}\left(\mathrm{CDCl}_{3}, 400 \mathrm{MHz}\right) \delta$ 7.40-7.35 (m, 2H), 6.91-6.87 (m, 2H), $4.83(\mathrm{~s}, 1 \mathrm{H}), 3.81(\mathrm{~s}$, $3 \mathrm{H}), 3.71(\mathrm{~s}, 3 \mathrm{H}), 3.61(\mathrm{ddd}, J=10.2 \mathrm{~Hz}, 9.2 \mathrm{~Hz}, 6.4 \mathrm{~Hz}, 1 \mathrm{H}), 3.52(\mathrm{ddd}, J=10.2 \mathrm{~Hz}, 9.2$ $\mathrm{Hz}, 6.2 \mathrm{~Hz}, 1 \mathrm{H}$ ), 1.05 (ddd, $J=13.8 \mathrm{~Hz}, 10.2 \mathrm{~Hz}, 6.4 \mathrm{~Hz}, 1 \mathrm{H}), 1.01$ (ddd, $J=13.8 \mathrm{~Hz}, 10.2$ $\mathrm{Hz}, 6.2 \mathrm{~Hz}, 1 \mathrm{H}), 0.01(\mathrm{~s}, 9 \mathrm{H})$.

${ }^{13} \mathrm{C} \mathrm{NMR}\left(\mathrm{CDCl}_{3}, 100 \mathrm{MHz}\right) \delta$ 172.0, 160.0, 129.1, 128.7, 114.2, 80.3, 67.2, 55.5, 52.4, 18.3,-1.2.

IR (film) 3001, 2953, 2896, 1753, 1611, 1513, 1249, 1172, 1100, 1035, 860, $837 \mathrm{~cm}^{-1}$.

MS (ESI) calcd for $\mathrm{C}_{15} \mathrm{H}_{24} \mathrm{NaO}_{4} \mathrm{Si}\left(\mathrm{M}^{+}+\mathrm{Na}\right)$ 319.1, found 319.0.

Methyl $\alpha$-4-acetaminophenyl- $\alpha$-(2-trimethylsilylethoxy)acetate (Table 3, entry 9). The compound was prepared according to the General Procedure using methyl $\alpha$-diazo$\alpha$-4-(acetaminophenyl)acetate $(93.3 \mathrm{mg}$ ). After chromatography on silica gel (hexanes/EtOAc/ $\mathrm{Et}_{3} \mathrm{~N}$ 66:34:0.3), the title compound was isolated as a colorless solid: run 1: $116 \mathrm{mg}(90 \%$; $89 \%$ ee); run 2: $111 \mathrm{mg}(88 \%$; $85 \%$ ee).

The ee was determined via HPLC on an OD-H column (hexanes/iso-propanol 93:7, flow $1.0 \mathrm{~mL} / \mathrm{min}$ ) with $\mathrm{t}_{\mathrm{r}}$ (major) $15.6 \mathrm{~min}, \mathrm{t}_{\mathrm{r}}$ (minor) $18.6 \mathrm{~min}$.

After a single crystalization from hexanes/EtOAc the title compound could be obtained in $99 \%$ ee.

mp: $135^{\circ} \mathrm{C}$;

$[\alpha]^{23}=77.4\left(\mathrm{c}=1.00, \mathrm{CHCl}_{3}\right)$.

${ }^{1} \mathrm{H} \mathrm{NMR}\left(\mathrm{CDCl}_{3}, 400 \mathrm{MHz}\right) \delta 7.50(\mathrm{~d}, J=8.6 \mathrm{~Hz}, 2 \mathrm{H}), 7.47(\mathrm{~s}, 1 \mathrm{H}), 7.39(\mathrm{~d}, J=8.6 \mathrm{~Hz}$, 2H), $4.85(\mathrm{~s}, 1 \mathrm{H}), 3.70(\mathrm{~s}, 3 \mathrm{H}), 3.61(\mathrm{ddd}, J=10.2 \mathrm{~Hz}, 9.2 \mathrm{~Hz}, 6.3 \mathrm{~Hz}, 1 \mathrm{H}), 3.52(\mathrm{ddd}, J=$ $10.2 \mathrm{~Hz}, 9.2 \mathrm{~Hz}, 6.3 \mathrm{~Hz}, 1 \mathrm{H}), 2.17(\mathrm{~s}, 3 \mathrm{H}), 1.04(\mathrm{ddd}, J=13.8 \mathrm{~Hz}, 10.2 \mathrm{~Hz}, 6.3 \mathrm{~Hz}, 1 \mathrm{H}), 1.00$ (ddd, $J=13.8 \mathrm{~Hz}, 10.2 \mathrm{~Hz}, 6.3 \mathrm{~Hz}, 1 \mathrm{H}), 0.00(\mathrm{~s}, 9 \mathrm{H})$.

${ }^{13} \mathrm{C}$ NMR $\left(\mathrm{CDCl}_{3}, 100 \mathrm{MHz}\right) \delta 171.8,168.6,138.4,132.7,128.1,120.1,80.3,67.4,52.4$, $24.8,18.3,-1.2$.

IR (film) 3304, 3066, 2953, 2896, 1746, 1670, 1605, 1555, 1411, 1249, 1211, 1173, 1105, $862,835 \mathrm{~cm}^{-1}$.

MS (ESI) calcd for $\mathrm{C}_{16} \mathrm{H}_{25} \mathrm{NNaO}_{4} \mathrm{Si}\left(\mathrm{M}^{+}+\mathrm{Na}\right)$ 346.2, found 345.9.

Methyl $\alpha$-biphenyl-4-yl- $\alpha$-(2-trimethylsilylethoxy)acetate (Table 3, entry 10). The compound was prepared according to the General Procedure using methyl $\alpha$-diazo- $\alpha$ (biphenyl-4-yl)acetate (101.0 mg). After chromatography on silica gel (hexanes / EtOAc 20:1), the title compound was isolated as a colorless oil: run 1: $128 \mathrm{mg}(93 \% ; 86 \%$ ee); run 2: $122 \mathrm{mg}$ (89\%; 85\% ee).

The ee was determined after cleavage of the trimethylsilylethyl group via HPLC on an OD-H column (hexanes/ iso-propanol 90:10, flow $1.0 \mathrm{~mL} / \mathrm{min}$ ) with $\mathrm{t}_{\mathrm{r}}$ (major) 10.6 min, $\mathrm{t}_{\mathrm{r}}($ minor) $12.2 \mathrm{~min}$.

$[\alpha]^{23}=77.9\left(\mathrm{c}=1.00, \mathrm{CHCl}_{3}\right)$.

${ }^{1} \mathrm{H}$ NMR $\left(\mathrm{CDCl}_{3}, 400 \mathrm{MHz}\right) \delta$ 7.63-7.58 (m, 4H), 7.56-7.52 (m, 2H), 7.48-7.42 (m, 2H), 7.39-7.34 (m, 1H), $4.95(\mathrm{~s}, 1 \mathrm{H}), 3.75(\mathrm{~s}, 3 \mathrm{H}), 3.69(\mathrm{ddd}, J=10.2 \mathrm{~Hz}, 9.2 \mathrm{~Hz}, 6.4 \mathrm{~Hz}, 1 \mathrm{H})$, $3.60(\mathrm{ddd}, J=10.2 \mathrm{~Hz}, 9.2 \mathrm{~Hz}, 6.2 \mathrm{~Hz}, 1 \mathrm{H}), 1.10(\mathrm{ddd}, J=13.9 \mathrm{~Hz}, 10.2 \mathrm{~Hz}, 6.4 \mathrm{~Hz}, 1 \mathrm{H})$, $1.06(\mathrm{ddd}, J=13.9 \mathrm{~Hz}, 10.2 \mathrm{~Hz}, 6.2 \mathrm{~Hz}, 1 \mathrm{H}), 0.04(\mathrm{~s}, 9 \mathrm{H})$. 
${ }^{13} \mathrm{C}$ NMR $\left(\mathrm{CDCl}_{3}, 100 \mathrm{MHz}\right) \delta$ 171.8, 141.7, 140.8, 135.9, 129.0, 127.7, 127.6, 127.5, 127.3, 80.6, 67.6, 52.5, 18.4, -1.2 .

IR (film) 3058, 3031, 2952, 2894, 1754, 1488, 1249, 1212, 1171, 1102, 1009, 860, 838, 758 $\mathrm{cm}^{-1}$.

MS (ESI) calcd for $\mathrm{C}_{20} \mathrm{H}_{26} \mathrm{NaO}_{3} \mathrm{Si}\left(\mathrm{M}^{+}+\mathrm{Na}\right)$ 365.2, found 365.0.

Ethyl $\alpha$-4-bromophenyl- $\alpha$-(2-trimethylsilylethoxy)acetate (Table 3, entry 11). The compound was prepared according to the General Procedure using ethyl $\alpha$-diazo- $\alpha-4-$ (bromophenyl)acetate (107.6 mg). After chromatography on silica gel (hexanes/EtOAc 20:1), the title compound was isolated as a colorless oil: run 1: $137 \mathrm{mg}(97 \% ; 78 \%$ ee); run 2: $132 \mathrm{mg}$ (92\%; 80\% ee).

The ee was determined after cleavage of the trimethylsilylethyl group via HPLC on an OD-H column (hexanes/iso-propanol 90:10, flow $1.0 \mathrm{~mL} / \mathrm{min}$ ) with $\mathrm{t}_{\mathrm{r}}$ (major) $6.9 \mathrm{~min}$, $\mathrm{t}_{\mathrm{r}}$ (minor) $7.6 \mathrm{~min}$.

$[\alpha]_{\mathrm{D}}^{23}=36.3\left(\mathrm{c}=1.00, \mathrm{CHCl}_{3}\right)$.

${ }^{1} \mathrm{H}$ NMR $\left(\mathrm{CDCl}_{3}, 400 \mathrm{MHz}\right)$ 8 7.51-7.48 (m, 2H), 7.36-7.33 (m, 2H), $4.82(\mathrm{~s}, 1 \mathrm{H}), 4.18$ $(\mathrm{m}, 2 \mathrm{H}), 3.65(\mathrm{ddd}, J=10.0 \mathrm{~Hz}, 9.1 \mathrm{~Hz}, 6.5 \mathrm{~Hz}, 1 \mathrm{H}), 3.54(\mathrm{ddd}, J=10.0 \mathrm{~Hz}, 9.1 \mathrm{~Hz}, 6.3 \mathrm{~Hz}$, $1 \mathrm{H}), 1.22(\mathrm{t}, J=7.1 \mathrm{~Hz}, 3 \mathrm{H}), 1.06(\mathrm{ddd}, J=13.9 \mathrm{~Hz}, 10.0 \mathrm{~Hz}, 6.5 \mathrm{~Hz}, 1 \mathrm{H}), 1.01$ (ddd, $J=$ $13.9 \mathrm{~Hz}, 10.0 \mathrm{~Hz}, 6.3 \mathrm{~Hz}, 1 \mathrm{H}), 0.02(\mathrm{~s}, 9 \mathrm{H})$.

${ }^{13} \mathrm{C}$ NMR $\left(\mathrm{CDCl}_{3}, 100 \mathrm{MHz}\right) \delta$ 170.9, 136.3, 131.9, 128.9, 122.7, 80.3, 67.6, 61.5, 18.4, 14.3,-1.2.

IR (film) 3054, 2954, 2895, 1751, 1487, 1250, 1177, 1122, 1096, 1013, 860, $838 \mathrm{~cm}^{-1}$.

MS (ESI) calcd for $\mathrm{C}_{15} \mathrm{H}_{23} \mathrm{BrNaO}_{3} \mathrm{Si}\left(\mathrm{M}^{+}+\mathrm{Na}\right) 381.1$, found 380.9 .

Methyl $\alpha$-4-fluorophenyl- $\alpha$-(2-trimethylsilylethoxy)acetate (Table 3, entry 12). The compound was prepared according to the General Procedure using methyl $\alpha$-diazo- $\alpha-4$ (fluorophenyl)acetate $(77.7 \mathrm{mg})$. After chromatography on silica gel (hexanes / EtOAc 20:1), the title compound was isolated as a colorless oil: run 1: $105 \mathrm{mg}(92 \% ; 89 \%$ ee); run 2: $103 \mathrm{mg}$ (91\%; 88\% ee).

The ee was determined after cleavage of the trimethylsilylethyl group via HPLC on an OD-H column (hexanes/iso-propanol 90:10, flow $1.0 \mathrm{~mL} / \mathrm{min}$ ) with $\mathrm{t}_{\mathrm{r}}$ (major) $7.6 \mathrm{~min}$, $\mathrm{t}_{\mathrm{r}}($ minor) $8.8 \mathrm{~min}$.

$[\alpha]^{23}=61.2\left(\mathrm{c}=1.00, \mathrm{CHCl}_{3}\right)$.

${ }^{1} \mathrm{H}$ NMR $\left(\mathrm{CDCl}_{3}, 400 \mathrm{MHz}\right)$ 8 7.46-7.41 (m, 2H), 7.08-7.02 (m, 2H), $4.86(\mathrm{~s}, 1 \mathrm{H}), 3.72(\mathrm{~s}$, $3 \mathrm{H}), 3.64(\mathrm{ddd}, J=10.2 \mathrm{~Hz}, 9.2 \mathrm{~Hz}, 6.3 \mathrm{~Hz}, 1 \mathrm{H}), 3.53(\mathrm{ddd}, J=10.3 \mathrm{~Hz}, 9.2 \mathrm{~Hz}, 6.1 \mathrm{~Hz}$, 1H), $1.07(\mathrm{ddd}, J=13.9 \mathrm{~Hz}, 10.3 \mathrm{~Hz}, 6.3 \mathrm{~Hz}, 1 \mathrm{H}), 1.00(\mathrm{ddd}, J=13.9 \mathrm{~Hz}, 10.2 \mathrm{~Hz}, 6.1 \mathrm{~Hz}$, $1 \mathrm{H}), 0.00(\mathrm{~s}, 9 \mathrm{H})$.

${ }^{13} \mathrm{C} \mathrm{NMR}\left(\mathrm{CDCl}_{3}, 100 \mathrm{MHz}\right) \delta$ 171.7, $163.0(\mathrm{~d}, J=247 \mathrm{~Hz}), 132.8(\mathrm{~d}, J=3.1 \mathrm{~Hz}), 129.1$ $(\mathrm{d}, J=8.4 \mathrm{~Hz}), 115.7(\mathrm{~d}, J=21.8 \mathrm{~Hz}), 80.1,67.6,52.5,18.4,-1.2$.

IR (film) 3074, 2954, 2895, 1755, 1605, 1509, 1250, 1224, 1173, 1114, 1014, 860, $838 \mathrm{~cm}^{-1}$. MS (ESI) calcd for $\mathrm{C}_{14} \mathrm{H}_{21} \mathrm{FNaO}_{3} \mathrm{Si}\left(\mathrm{M}^{+}+\mathrm{Na}\right)$ 307.1, found 307.0.

Methyl $\alpha$-4-trifluoromethylphenyl- $\alpha$-(2-trimethylsilylethoxy)acetate (Table 3, entry 13). The compound was prepared according to the General Procedure using 
methyl $\alpha$-diazo- $\alpha$-4-(trifluoromethylphenyl)acetate $(97.7 \mathrm{mg}$ ). After chromatography on silica gel (hexanes/EtOAc 20:1), the title compound was isolated as a colorless oil: run 1: $121 \mathrm{mg}$ (90\%; 19\% ee); run 2: $119 \mathrm{mg}(89 \% ; 22 \%$ ee).

The ee was determined after cleavage of the trimethylsilylethyl group via HPLC on an OD-H column (hexanes/iso-propanol 97:3, flow $1.0 \mathrm{~mL} / \mathrm{min}$ ) with $\mathrm{t}_{\mathrm{r}}$ (major) $14.0 \mathrm{~min}$, $\mathrm{t}_{\mathrm{r}}$ (minor) $15.5 \mathrm{~min}$.

$[\alpha]^{23}=10.6\left(\mathrm{c}=1.03, \mathrm{CHCl}_{3}\right)$.

${ }^{1} \mathrm{H}$ NMR $\left(\mathrm{CDCl}_{3}, 400 \mathrm{MHz}\right) \delta 7.64-7.59(\mathrm{~m}, 4 \mathrm{H}), 4.95(\mathrm{~s}, 1 \mathrm{H}), 3.73(\mathrm{~s}, 3 \mathrm{H}), 3.69(\mathrm{ddd}, J=$ $10.2 \mathrm{~Hz}, 9.1 \mathrm{~Hz}, 6.3 \mathrm{~Hz}, 1 \mathrm{H}), 3.56$ (ddd, $J=10.2 \mathrm{~Hz}, 9.1 \mathrm{~Hz}, 6.1 \mathrm{~Hz}, 1 \mathrm{H}), 1.08$ (ddd, $J=$ $13.9 \mathrm{~Hz}, 10.2 \mathrm{~Hz}, 6.3 \mathrm{~Hz}, 1 \mathrm{H}), 1.03$ (ddd, $J=13.9 \mathrm{~Hz}, 10.2 \mathrm{~Hz}, 6.1 \mathrm{~Hz}, 1 \mathrm{H}), 0.02(\mathrm{~s}, 9 \mathrm{H})$.

${ }^{13} \mathrm{C}$ NMR $\left(\mathrm{CDCl}_{3}, 100 \mathrm{MHz}\right) \delta$ 171.2, 140.9, $138.9(\mathrm{q}, J=32.5 \mathrm{~Hz}), 127.5,125.7(\mathrm{q}, J=3.8$ $\mathrm{Hz}), 124.2(\mathrm{q}, J=272 \mathrm{~Hz}), 80.3,68.0,52.6,18.4,-1.2$.

IR (film) 3053, 2956, 2896, 1758, 1419, 1327, 1251, 1168, 1128, 1068, 860, $838 \mathrm{~cm}^{-1}$.

MS (ESI) calcd for $\mathrm{C}_{15} \mathrm{H}_{20} \mathrm{~F}_{3} \mathrm{O}_{3} \mathrm{Si}\left(\mathrm{M}^{+}-\mathrm{H}+\mathrm{Na}\right)$ 333.1, found 333.0.

Methyl $\alpha$-naphthalene-2-yl- $\alpha$-(2-trimethylsilylethoxy)acetate (Table 3, entry 14). The compound was prepared according to the General Procedure using methyl $\alpha$-diazo$\alpha$-(naphthalene-2-yl)acetate $(95.5 \mathrm{mg})$. After chromatography on silica gel (hexanes/EtOAc 18:1), the title compound was isolated as a colorless oil: run 1: $119 \mathrm{mg}$ (94\%; 84\% ee); run 2: $116 \mathrm{mg}(92 \% ; 83 \%$ ee).

The ee was determined after cleavage of the trimethylsilylethyl group via HPLC on an OD-H column (hexanes/iso-propanol 90:10, flow $1.0 \mathrm{~mL} / \mathrm{min}$ ) with $\mathrm{t}_{\mathrm{r}}$ (major) 12.1 min, $\mathrm{t}_{\mathrm{r}}$ (minor) $14.2 \mathrm{~min}$.

$[\alpha]_{D}^{23}=86.9\left(\mathrm{c}=0.99, \mathrm{CHCl}_{3}\right)$.

${ }^{1} \mathrm{H}$ NMR $\left(\mathrm{CDCl}_{3}, 400 \mathrm{MHz}\right) \delta$ 7.95-7.93 (m, 1H), 7.89-7.82 (m, 3H), 7.61-7.58 (m, $\left.1 \mathrm{H}\right)$, 7.53-7.47 (m, 2H), $5.07(\mathrm{~s}, 1 \mathrm{H}), 3.73(\mathrm{~s}, 3 \mathrm{H}), 3.70(\mathrm{ddd}, J=10.2 \mathrm{~Hz}, 9.2 \mathrm{~Hz}, 6.3 \mathrm{~Hz}, 1 \mathrm{H})$, $3.61(\mathrm{ddd}, J=10.2 \mathrm{~Hz}, 9.2 \mathrm{~Hz}, 6.3 \mathrm{~Hz}, 1 \mathrm{H}), 1.11(\mathrm{ddd}, J=13.9 \mathrm{~Hz}, 10.2 \mathrm{~Hz}, 6.3 \mathrm{~Hz}, 1 \mathrm{H})$, 1.08 (ddd, $J=13.9 \mathrm{~Hz}, 10.2 \mathrm{~Hz}, 6.3 \mathrm{~Hz}, 1 \mathrm{H}), 0.03$ (s, 9H).

${ }^{13} \mathrm{C}$ NMR $\left(\mathrm{CDCl}_{3}, 100 \mathrm{MHz}\right) \delta 171.8,134.4,133.6,133.3,128.6,128.3,127.9,126.8$, $126.52,126.47,124.8,80.9,67.5,52.5,18.4,-1.2$.

IR (film) 3058, 2952, 2894, 1754, 1435, 1249, 1198, 1176, 1108, 859, 839, $753 \mathrm{~cm}^{-1}$.

MS (ESI) calcd for $\mathrm{C}_{18} \mathrm{H}_{24} \mathrm{NaO}_{3} \mathrm{Si}\left(\mathrm{M}^{+}+\mathrm{Na}\right) 339.2$, found 339.0.

\section{Methyl $\alpha$-benzo[1,3]dioxol-5-yl- $\alpha$-(2-trimethylsilylethoxy)acetate (Table 3, entry} 15). The compound was prepared according to the General Procedure using methyl $\alpha$ diazo- $\alpha$-(benzo[1,3]dioxol-5-yl)acetate $(88.1 \mathrm{mg})$. After chromatography on silica gel (hexanes/EtOAc 15:1), the title compound was isolated as a colorless oil: run 1: $110 \mathrm{mg}$ (89\%; 89\% ee); run 2: $107 \mathrm{mg}(87 \%$; $88 \%$ ee).

The ee was determined via HPLC on an OD-H column (hexanes/iso-propanol 99:1, flow $1.0 \mathrm{~mL} / \mathrm{min}$ ) with $\mathrm{t}_{\mathrm{r}}$ (major) $6.1 \mathrm{~min}, \mathrm{t}_{\mathrm{r}}(\mathrm{minor}) 7.3 \mathrm{~min}$.

$[\alpha]^{23}=73.6\left(\mathrm{c}=1.01, \mathrm{CHCl}_{3}\right)$.

${ }^{1} \mathrm{H}$ NMR $\left(\mathrm{CDCl}_{3}, 400 \mathrm{MHz}\right) \delta 6.96(\mathrm{~d}, J=1.7 \mathrm{~Hz}, 1 \mathrm{H}), 6.91(\mathrm{dd}, J=7.9 \mathrm{~Hz}, 1.7 \mathrm{~Hz}, 1 \mathrm{H})$, $6.78(\mathrm{~d}, J=7.9 \mathrm{~Hz}, 1 \mathrm{H}), 5.97(\mathrm{~m}, 2 \mathrm{H}), 4.79(\mathrm{~s}, 1 \mathrm{H}), 3.72(\mathrm{~s}, 3 \mathrm{H}), 3.59(\mathrm{ddd}, J=10.3 \mathrm{~Hz}, 9.2$ 
$\mathrm{Hz}, 6.4 \mathrm{~Hz}, 1 \mathrm{H}), 3.52(\mathrm{ddd}, J=10.3 \mathrm{~Hz}, 9.2 \mathrm{~Hz}, 6.2 \mathrm{~Hz}, 1 \mathrm{H}), 1.05(\mathrm{ddd}, J=13.8 \mathrm{~Hz}, 10.3$

$\mathrm{Hz}, 6.4 \mathrm{~Hz}, 1 \mathrm{H}$ ), 1.00 (ddd, $J=13.8 \mathrm{~Hz}, 10.3 \mathrm{~Hz}, 6.2 \mathrm{~Hz}, 1 \mathrm{H}), 0.00$ (s, 9H).

${ }^{13} \mathrm{C} \mathrm{NMR}\left(\mathrm{CDCl}_{3}, 100 \mathrm{MHz}\right) \delta 171.8,148.09,148.05,130.8,121.2,108.4,107.7,101.4$, $80.5,67.3,52.4,18.3,-1.2$.

IR (film) 3075, 2953, 2894, 1753, 1504, 1490, 1445, 1248, 1201, 1172, 1103, 1040, 933, 861, $839 \mathrm{~cm}^{-1}$.

MS (ESI) calcd for $\mathrm{C}_{15} \mathrm{H}_{22} \mathrm{NaO}_{5} \mathrm{Si}\left(\mathrm{M}^{+}+\mathrm{Na}\right)$ 333.1, found 333.0.

Methyl $\alpha$-thiophene-3-yl- $\alpha$-(2-trimethylsilylethoxy)acetate (Table 3, entry 16). The compound was prepared according to the General Procedure using methyl $\alpha$-diazo- $\alpha$ (thiophene-3-yl)acetate $(72.9 \mathrm{mg})$. After chromatography on silica gel (hexanes $/ \mathrm{EtOAc} / \mathrm{Et}_{3} \mathrm{~N}$ 25:1:0.1), the title compound was isolated as a colorless oil: run 1: $97 \mathrm{mg}$ (89\%; 89\% ee); run 2: $95 \mathrm{mg}(87 \%$; 87\% ee).

The ee was determined after cleavage of the trimethylsilylethylgroup via HPLC on an OD-H column (hexanes/iso-propanol 95:5, flow $1.0 \mathrm{~mL} / \mathrm{min}$ ) with $\mathrm{t}_{\mathrm{r}}$ (major) $13.7 \mathrm{~min}$, $\mathrm{t}_{\mathrm{r}}$ (minor) $17.3 \mathrm{~min}$.

$[\alpha]^{23}=51.8\left(\mathrm{c}=1.01, \mathrm{CHCl}_{3}\right)$.

${ }^{1} \mathrm{H}$ NMR $\left(\mathrm{CDCl}_{3}, 400 \mathrm{MHz}\right) \delta$ 7.37-7.35 (m, $\left.1 \mathrm{H}\right), 7.30(\mathrm{dd}, J=5.0 \mathrm{~Hz}, J=3.0 \mathrm{~Hz}, 1 \mathrm{H})$, $7.15(\mathrm{dd}, J=5.0 \mathrm{~Hz}, J=1.3 \mathrm{~Hz}, 1 \mathrm{H}), 5.00(\mathrm{~s}, 1 \mathrm{H}), 3.75(\mathrm{~s}, 3 \mathrm{H}), 3.65(\mathrm{ddd}, J=10.1 \mathrm{~Hz}, 9.1$ $\mathrm{Hz}, 6.6 \mathrm{~Hz}, 1 \mathrm{H}), 3.56(\mathrm{ddd}, J=10.1 \mathrm{~Hz}, 9.1 \mathrm{~Hz}, 6.4 \mathrm{~Hz}, 1 \mathrm{H}), 1.05(\mathrm{ddd}, J=13.9 \mathrm{~Hz}, 10.1$ $\mathrm{Hz}, 6.6 \mathrm{~Hz}, 1 \mathrm{H}), 1.02(\mathrm{ddd}, J=13.9 \mathrm{~Hz}, 10.1 \mathrm{~Hz}, 6.4 \mathrm{~Hz}, 1 \mathrm{H}), 0.02(\mathrm{~s}, 9 \mathrm{H})$.

${ }^{13} \mathrm{C} \mathrm{NMR}\left(\mathrm{CDCl}_{3}, 100 \mathrm{MHz}\right) \delta$ 171.5, 137.6, 126.4, 126.3, 123.6, 77.1, 67.6, 52.5, 18.3, 1.21 .

IR (film) 3106, 2953, 2894, 1755, 1436, 1249, 1198, 1174, 1107, 860, 839, $773 \mathrm{~cm}^{-1}$.

MS (ESI) calcd for $\mathrm{C}_{12} \mathrm{H}_{20} \mathrm{NaO}_{3} \mathrm{SSi}\left(\mathrm{M}^{+}+\mathrm{Na}\right)$ 295.1, found 295.0.

Eq 3. In a glove box, $\mathrm{Cu}(\mathrm{OTf})_{2}(0.6 \mathrm{mg}, 1.6 \mu \mathrm{mol})$ and $(+)-1(1.6 \mathrm{mg}, 3.0 \mu \mathrm{mol})$ were dissolved in 1,2-dichloroethane $(200 \mu \mathrm{L})$ that contained water $(3.2 \mu \mathrm{mol} ; 50 \% \mathrm{D})$. The resulting mixture was stirred for $20 \mathrm{~min}$ at $\mathrm{rt}$, and then it was passed through an acrodisc filter and added in one portion to a solution of methyl $\alpha$-diazo- $\alpha$-phenylacetate (14.1 mg, $0.080 \mathrm{mmol})$ and $\mathrm{TMSCH}_{2} \mathrm{CH}_{2} \mathrm{OH}(\mathrm{D})(46 \mu \mathrm{L}, 12.2 \mathrm{mg}, 0.32 \mathrm{mmol} ; 50 \% \mathrm{D}$ according to $\left.{ }^{1} \mathrm{H} \mathrm{NMR}\right)$ in 1,2-dichloroethane $(8 \mathrm{~mL})$. The reaction mixture was stirred for $1.0 \mathrm{~h}$ at rt. Then, the solvent was evaporated, the residue was passed through a plug of silica gel ( $\mathrm{Et}_{2} \mathrm{O}$ as eluent), and the deuterium incorporation was determined by NMR. Run 1: 28\% D (99\% yield); run 2: 27\% D (98\% yield). 


\section{References}

(1) Lo, M. M.-C.; Fu, G. C. J. Am. Chem. Soc. 1998, 120, 10270-10271.

(2) Effenberger, F.; Hopf, M.; Ziegler, T.; Hudelmayer, J. Chem. Ber. 1991, 124, 16511659.

(3) Shintou, T.; Mukaiyama, T. J. Am. Chem. Soc. 2004, 126, 7359-7367.

(4) Shintou, T.; Mukaiyama, T. J. Am. Chem. Soc. 2004, 126, 7359-7367. 